\title{
Developments of calcium phosphate-based bone regenerating materials utilizing interfacial interactions between inorganic-organic substances
}

\author{
Masanori KIKUCHI ${ }^{1, \dagger}$ \\ ${ }^{1}$ Bioceramies Group, National Institute for Materials Science, 1-1 Namiki, Tsukuba, Ibaraki 305-0044, Japan
}

\begin{abstract}
Calcium phosphate-based bone regenerating materials were developed by utilizing interfacial interaction between inorganic-organic substances. Although apatitic calcium phosphates, hydroxyapatite (HAp) and $\beta$ tricalcium phosphate $(\beta$-TCP), have unique affinity to organic substances, and utilization of the affinity requires appropriate surrounding conditions. The author and his colleagues control the surrounding conditions to realize porous HAp ceramics with high porosity, interconnectivity and compressive strength, composite membrane of $\beta$ TCP and polylactide-based biodegradable polymers for guided bone regeneration, and bone-like nanocomposite of HAp and type-I atelocollagen (HAp/Col). Electrostatic interactions between calcium phosphates and polymers in these composites were presented by reflection infrared spectra. They also examined in vitro and in vivo and demonstrate good bone regeneration properties. Particularly, the HAp/Col exhibits completely incorporation into bone remodeling process that is the first in the world for synthetic materials. Three of these materials are also commercialized and used in medical and dental fields and contribute to human health. (c2020 The Ceramic Society of Japan. All rights reserved.
\end{abstract}

Key-words : Calcium phosphates, Interfacial interaction, Binder, Organic polymer, Bone tissue reaction

\section{Introduction}

Apatitic calcium phosphates, hydroxyapatite $\left[\mathrm{Ca}_{10^{-}}\right.$ $\left.\left(\mathrm{PO}_{4}\right)_{6}(\mathrm{OH})_{2}, \mathrm{HAp}\right]$ and tricalcium phosphate, $\left[\mathrm{Ca}_{3}\left(\mathrm{PO}_{4}\right)_{2}\right.$, TCP] of $\alpha$ - and $\beta$-phases, are widely used as bone void fillers due to their excellent biocompatibility, so-called bioactivity, a property to bond directly with bone. Further, these calcium phosphates have a unique property to adsorb biomolecules, nucleic acids, proteins, amino acids, saccharides and lipids. This property have been utilized as adsorbent of liquid chromatography and toothpaste to remove saccharides and other organic molecules from tooth surface effectively. However, not so many reports were found in utilization the affinity, in other words, interfacial interaction between organic substances and apatitic calcium phosphates to develop new materials.

In this review, research and development of three new bone regenerating materials by utilizing the interfacial interactions are described.

\section{Porous HAp ceramics}

In the 20th century, artificial bone void fillers composed of HAp have low porosity up to $65 \%$ due to the loss of mechanical strength by increasing their porosity. In fact,

\footnotetext{
Corresponding author: M. Kikuchi; E-mail: KIKUCHI. Masanori@nims.go.jp
}

orthopedic surgeons frequently push the bone void fillers into bone defect; thus, weak materials are not preferred by them. Many researchers tried to increase porosity to more than $75 \%$ without losing mechanical strength but did not succeed. The authors focused on the following two points to strengthen a porous body: 1 . Good sinterability to achieve high mechanical strength between granules as well as low pores (crucks) formation between granules, 2. Ignoring cruck formation during burning of porogens. The second requirement denied using polymeric porogen which inflates during burning process, and the expansion stretches pore wall to create crucks. Accordingly, foam casting is chosen for the preparation. The first requirement is divided into two parts. One is starting HAp powder and the other is a binder molecule. From the author's experiences, HAp prepared by a wet method using own-made $\mathrm{Ca}(\mathrm{OH})_{2}$ from highly pure $\mathrm{CaCO}_{3}$ (e.g., Alkaline analysis grade, Wako Pure chemical, Osaka, Japan) and $\mathrm{H}_{3} \mathrm{PO}_{4}$ (e.g., Reagent grade, Wako Pure chemical, Osaka, Japan) as starting materials shows good sinterability more than $99.5 \%$ of relative density when appropriate organic binder (e.g., polyvinyl alcohol) is used. Therefore, finding appropriate binder to prepare foam and crosslinker to fix a porous structure is important issue for fabricate novel porous bone void filler having $75 \%$ porosity with fully interconnected pores and the same compressive strength as the conventional bone void fillers, approximately $10 \mathrm{MPa}$. 
This work was performed in collaboration with Toshiba Ceramics Co. Itd (at that time, CoorsTek Inc. in present) that have good procedure to fabricate foam-casting of alumina ceramics. After the transfer of the HAp symthesis method from the author, they still faced a problem in sinterability of HAp by using their conventional binder and crosslinker. The author hypothesized that the problem could be attributed to the interaction between a binder and the HAp surface. Generally, the HAp synthesized by our method and calcined at $800^{\circ} \mathrm{C}$ has a negative surface charge. Thus, a binder with positive functional group, such as amino and imino groups, could interact to the HAp surface and could play a role of good binder. A binder and crosslinker chosen by the hypothesis worked as expected and the authors succeeds to prepare HAp porous ceramics with $75 \%$ porosity, high compressive strength and high interconnected porous structure. ${ }^{1)}$ This porous HAp was then moved to animal experiments and clinical trial collaborated with Department of Orthopedic Surgery of Osaka University Medical School, National Institute of Advanced Industrial Science and Technology and MMT Co. Ltd. (at that time, Aimedic MMT Co. Ltd. in present). The porous HAp was then commercialized as Neobone ${ }^{\circledR}$ in 2003.

Similar approach was also applied to fabricate uniaxial porous body of HAp using ice crystal growth in HAp containing hydrogel, which was sold as Regenos ${ }^{\circledR}$. ${ }^{2)}$

\section{Composite with synthetic biodegradable polymers}

Although biocomaptibility and bioactivity of apatitic calcium phosphates are excellent in comparison to other materials, a high elastic modulus, cause of stress shielding (reason of inhibition of bone maturation), and brittleness, cause of fracture, are still problems on practical use. Ceramics with higher porosity, as mentioned in the section 2 , reduce those risks but remaining of ceramics with different mechanical properties to bone have a potential risk of fractures in the materials and/or on the interface between bone and ceramics. The author considered that this problem can be solved by the following two ways. One is using a inorganic/organic composite that has a similar mechanical properties, and the other is using a biodegradable material that degrades with formation (regeneration) of novel bone tissue; however, both ways have respective problems. Big problem on the former one is fatigue of material which reduce mechanical properties and increase a risk of fracture. In fact, HAPEX ${ }^{\circledR}$, a composite of HAp and polyethylene, developed by Bonfiled and his colleagues demonstrated bone-like mechanical properties and bioactivity $^{3)}$ but is only applied to non-load-bearing site ${ }^{4)}$ to reduce the risk of fracture caused by the material. As for the latter one, the most famous biodegradable ceramics at that time in Japan is Osferion ${ }^{\circledR}$, the first commercialized $\beta$ TCP bone void filler in Japan, from Olympus Corp. (at that time, Olympus Terumo Biomaterials Corp. in present). It generally needs about a year for complete degradation in practice; therefore, it may remain some risk of fracture after removing fixator.
Therefore, many researchers tried to prepare inorganic/ organic composite with biodegradability. At that date, many researchers had worked on a combination of synthetic biodegradable polymer and HAp, but degradation behavior of HAp in those kinds of composite is still unclear. On the other hand, poly $\alpha$ hydroxy acids represented by poly-L-lactic acid degrades by hydrolysis reaction at carbonyl group on its chain, and its degradation ratio is accelerated by humidity, temperature, basic and acidic conditions. This means that degradation of polymer is selfaccelerated by acid from degraded polymer, especially for the polymer of which decomposition products are low molecular weight, e.g., glycolic acid and lactic acid. In addition to that, acidic conditions induced by polymer degradation will cause of inflammation of surrounding tissues. Including this problem, poly $\alpha$ hydroxy acid has the following problems when it used for bone void filler, no osteoconductivity and low elastic modulus. The latter two issues had already considered by researchers studied on composites using HAp, acid problem had been little focused, at least no descriptions in any paper at that time.

The author considered all above problems and focused on combination of synthetic biodegradable polymer and $\beta$ TCP, obvious biodegradable ceramics. That is to say, $\beta$ TCP "ceramics" increases elastic modulus, $\beta$-TCP "bioactive (a material directly bond with bone)" ceramics supplements osteoconductivity (bone grows on the surface of a material) and $\beta$-TCP "biodegradable" bioactive ceramics compensates acidification, because $\beta$-TCP is a substance composed of weak acid and strong base. As synthetic polymers, the author had chosen two type of poly-Llactide based copolymers to control mechanical properties and degradation rate. One is copolymerized poly-L-lactide with sebacic acid (CPLA) and the other is poly-L-lactideco-glycolide-co- $\varepsilon$-caprolactone (PLGC). Essentially, these two polymers demonstrate similar results in physicochemical and biological properties of composites.

General preparation method for polymer/ceramics composite is a mixing of ceramics with a polymer dissolved in nonpolar solvent and volatilize solvent after casting. This is of course good method to reduce a risk of decomposition of poly $\alpha$ hydroxy acid. However, interaction between "polar" ceramics and "nonpolar" polymer is difficult to form by this method, even though HAp and $\beta$-TCP have a good affinity to some organic substances including lipids. In fact, gap is formed between $\beta$-TCP particles and the above polymers by the solvent mixing method. ${ }^{5)}$ This problem is solved by surface treatment of ceramics to improve affinity to nonpolar substances in structural composite, carbon and glass fiber reinforced plastic, but supplementation of the third substance should avoid as possible if the material will be used as a biomaterial. Therefore, the author changed the preparation way to a heat-kneading which mix $\beta$-TCP with molten poly $\alpha$ hydroxy acids melt at higher than melting points under vacuum. ${ }^{5), 6)}$ High temperature may decompose polymer but decomposition rate will be reduce by avoiding water in air and on ceramics surface. After the preparation, 


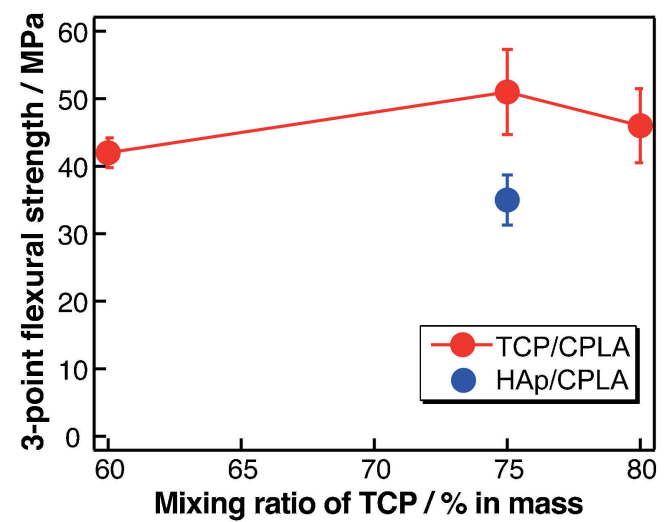

Fig. 1. Changes in three-point flexural strength as a function of mixing ratio of $\beta$-TCP in composite.

obtained composites were evaluated physicochemical and biological properties ex and in vivo.

Three-point flexural strengths of the for TCP/CPLA composites demonstrate in the range of $40-50 \mathrm{MPa}$ at different mixing ratios as shown in Fig. 1. ${ }^{6}$ These values are slight weaker than pure CPLA. Mass mixing ratio of $75 \%$ of $\beta$-TCP is very near to equal volume ratio between $\beta$ TCP and CPLA (precise mass ratio for that is $72-3 \%$ ) and shows the highest strength in trend, and as a comparison, a result of HAp/CPLA at the same mixing ratio and the same preparation method also is indicated in Fig. 1. Interestingly, three-point flexural strength of the HAp/CPLA drastically decreases to $70 \%$ in comparison to the TCP/ CPLA, because the weight average molecular weight of CPLA for the HAp/CPLA was much decreased from 150 to $60.3 \mathrm{kDa}$ than that of the TCP/CPLA of $110 \mathrm{kDa}$. The reason for the difference is considered to be the presence of hydroxyl groups in HAp, which would acted as a catalyst for polymer degradation during kneading at high temperature and promoted polymer degradation even under vacuum. ${ }^{6)}$ The TCP/CPLA and TCP/PLGC composites demonstrate good thermoplastic property above glass transition temperature of CPLAs ${ }^{6)}$ and PLGCs. ${ }^{5)}$ Further, scanning electron micrograph of fracture surface of the TCP/ CPLA shown in Fig. 2 exhibits homogeneous dispersion of $\beta$-TCP particles and good wettability between $\beta$-TCP particles and CPLA $^{6)}$ or PLGC, ${ }^{5)}$ which suggests a presence of physicochemical interaction between them.

The physicochemical interaction between $\beta$-TCP and CPLA was investigated by a reflection method using Fourier-transformed infrared (FTIR) spectrometer at 60, 75 and $85^{\circ}$ of reflection angle. Mixing ratios of the $\beta$-TCP and CPLA were 50, 75, 85 and $90 \%$. The reflectance spectra of FTIR were converted to absorbance spectra by the Kramers-Kronig method. The reflectance of particle filler type composite mirrors multiple reflection of interface between particle fillers and polymer. The energy for interaction has to be supplied from both substances; therefore, presence of physicochemical interaction is indicated as a red shift of absorption band of functional group in polymer reflecting energy consumption for the physicochemical

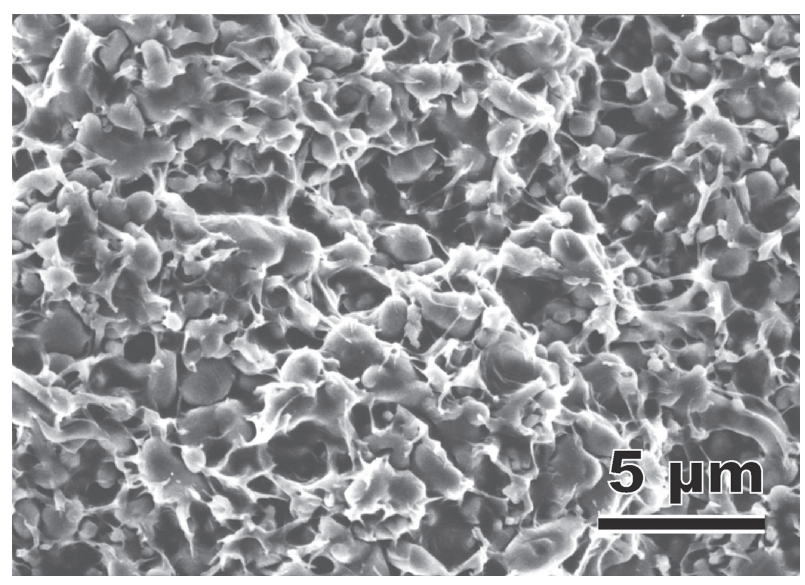

Fig. 2. Fracture surface of TCP/CPLA composite.

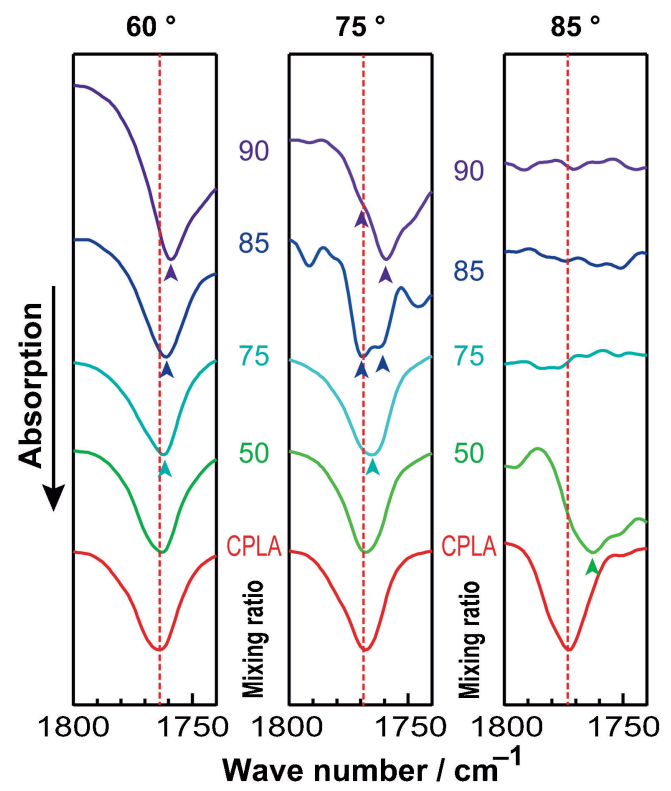

Fig. 3. Reflection FTIR spectra of TCP/CPLA composite.

interaction. The results summarized in Fig. 3 illustrate stretching band of carbonyl group in the CPLA observed around wave number of $1770 \mathrm{~cm}^{-1}$. Spectra of lower reflection angle, $60^{\circ}$, indicate no significant red shift due to small multiple reflection numbers; however, spectra of higher angles, 75 and $85^{\circ}$, depict red shift of stretching band of carbonyl group, including splitting in two bands in some case. ${ }^{7)}$ Unfortunately, this interaction which improves mechanical strength and thermoplasticity could become disadvantage in bioactivity, i.e., osteoconductivity, because $\beta$-TCP particles are mostly covered with the CPLA.

Changes in $\mathrm{pH}$ of physiological saline and phosphate buffered saline in which the composite was soaked demonstrate similar results. ${ }^{5), 8)}$ As expected at material design, $\mathrm{pH}$ maintains neutral during polymer decomposition.

No cytotoxic signs are found in cell culture tests of the composites. ${ }^{9)}$ After a preliminary animal experiment, significantly lower osteoconductivity than HAp and $\beta$-TCP is confirmed, even though the composite presents good apatite forming ability in simulated body fluid than HAp and 
$\beta$-TCP. ${ }^{10)}$ Therefore, in vivo tests using dogs to apply the composite membrane for guided bone regeneration (GBR) were performed. A GBR is one of guided tissue regenerations. Small tissue defect of human is generally filled with regenerated original tissue; however, defect size excesses tissue-dependent limit of regeneration, most of the defect is filled with scar tissue. This is a protective reaction of human body performed by invasion of fibroblasts, collagen fiber producing cells. Therefore, inhibition of fibroblasts migration by membrane supports original tissue reconstruction.

The authors tried to regenerate large bone defect on mandible and tibia of beagles. Buccal-lingual defects of $10 \times 10 \times 10 \mathrm{~mm}^{3}$, i.e., whole bone removal in the direction, were created on both sides of the alveolar bone of the mandible. One side was GBR treated by covering defect with a TCP/CPLA membrane fitted to the surrounding alveolar shape by warming in physiological saline of $50^{\circ} \mathrm{C}$ and the other side was a negative control, i.e., creation of defect without the GBR treatment, and both sides were closed by suturing the gingiva. Full segmental tibial defect of $20 \mathrm{~mm}$ in length was created after an application of external fixator and was GBR treated by the similar procedure to mandible. Other dog was used for negative controls, with the GBR treatment with the CPLA or without the GBR treatment of the same-sized tibial defect. All the animal tests described in the present review paper are conformed with "The Guidelines of Tokyo Medical and Dental University for Animal Care" based on "The NIH Guidelines for the Care and Use of Laboratory Animals" (NIH Publication \#85-23 Rev. 1985). All treatments were performed by veterinarians, whereas animal maintenance was performed by veterinarians and animal health technicians.

In mandible defects, the experimental group exhibits quite good regeneration that at least $90 \%$ of bone height is recovered at the center of defect after 12 weeks postoperation. Further, no sign of remaining of TCP/CPLA membrane is observed. Tibial defect GBR model also demonstrates good results that complete bridging between distal and proximal edges is observed with remaining of membrane at 12 weeks after operation; whereas GBR treatment with pure CPLA membrane and no GBR treatment do not achieve bone bridging at all. ${ }^{8)}$ The similar results are found for GBR treatment with the TCP/PLGC membrane. ${ }^{5)}$

\section{Bone-like nanocomposite}

\subsection{Composite toward real bone}

Vertebrates bone is a typical nanocomposite composed of approximately same volume of calcium deficient carbonate-containing HAp as an inorganic substance and type-I atelocollagen (Col, collagen that antigenic telopepitdes are enzymatically removed) as a dominant organic substance. In addition to that, bone has a nanostructure in which $c$-axes of HAp nanocrystals aligns elongation direction of Col fibers, the same as Col long axis. Therefore, these main components, HAp and Col are of course main target to fabricate composite for bone void fillers as well as elucidating bone formation mechanism for biomimetic synthesis of materials. For instance, Du et al., implanted mixture of nano-HAp and Col in marrow cavity. ${ }^{11)}$ Further, so-called "biomimetic" approaches are also performed by many researchers, i.e., HAp nanocrystals are formed on prefabricated $\mathrm{Col}$ fibers in sponges or membranes composed of Col. However, they have no bone-like nanostructures and demonstrates no similar bone tissue response to autologous bone transplantation, incorporating into bone remodeling process. The bone remodeling process is also observed in both remodeling old bone into new bone and bone maturation at bone fracture repair, and is osteoclastic "old" and/or "broken" bone resorption followed by osteoblastic new bone formation. The osteoclastic bone resorption is based on the following pure chemical reactions: acidification of a resorption site by supplying proton to dissolve HAp nanocrystals in bone and decomposition of Col by supplying enzymes. Bone formation by osteoblasts is also sequential chemical reactions: 1 . Secretion of $\mathrm{Col}$ as protocollagen, 2. Fibrillogenesis of $\mathrm{Col}$ and 3. Deposition of calcium phosphate (HAp or its precursors). On the contrary, ex vivo the "biomimetic" precipitation of HAp on Col fiber only showed limited formation of HAp in Col fibers and limited orientation of HAp nanocrystals on Col fibers when HAp mass ratio in composites becomes close to that in bone. In 2007, improved biomimetic calcification process, polymer-induced liquid precursor (PILP)" was proposed by Gower's group who works on biomimetic calcification processes of hard tissues, i.e., Olszta et al., fabricated bone-like composite of HAp and Col by precipitation of HAp in and on Col fibers utilizing polylysine as a liquid precursor. ${ }^{12)}$ The method is good to elucidate biomimetic process but not suit for mass production of bone void fillers.

\subsection{Simultaneous titration method to fabri- cate bone-like nanocomposite}

Prior to Olszta's work, the author and his colleagues reported a synthesis method and bone tissue reaction of bone-like nanocomposite of HAp and $\mathrm{Col}$ (HAp/Col) having bone-like nanostructure and chemical composition in 2001. ${ }^{13)}$ The author has not considered that osteoblasts align HAp nanocrystals and Col directly by their tentacles. In fact, size relation between HAp nanocrystals in bone, $20-40 \mathrm{~nm}$ in long direction, and cell tentacles is like relation of that between sand particles and human finger. Obviously, we cannot align sand particles in one crystallographic direction along sewing threads by our fingers; hence, cells must not be able align HAp nanocrystals and Col fibers by their tentacles, either. Nevertheless, HAp and $\mathrm{Col}$ are align each other in bone, supplying raw materials and controlling surroundin condition osteoblasts' role is enough to realize bone-like nanostructure. Therefore, the authors proposed the simultaneous titration method, a very simple system to mimic osteoblast roles. Briefly, raw materials, calcium hydroxide suspension and orthophosphoric acid solution containing Col, are simultaneously titrated through each tube pump into a reaction vessel in which the same amount of pure water as calcium hydroxide 


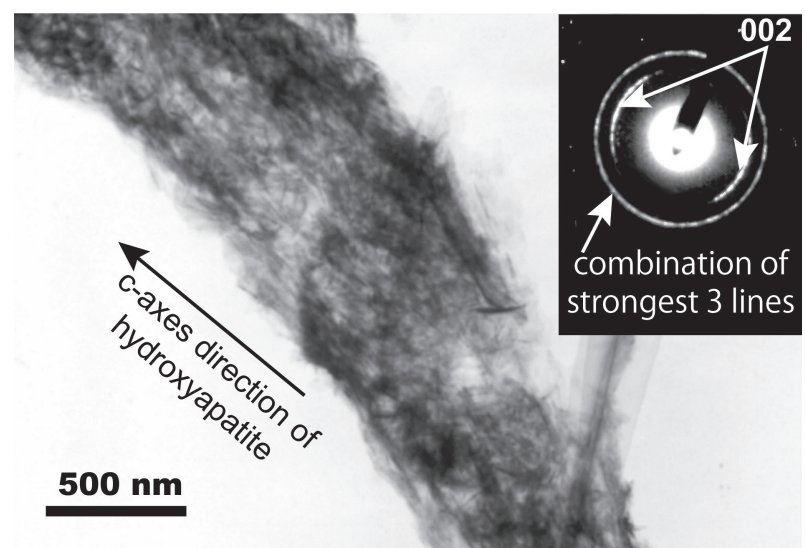

Fig. 4. Transmission electron micrograph of HAp/Col with selected area electron diffraction pattern.

Table 1. Synthesis conditions for HAp/Col for examination of influence of concentration

\begin{tabular}{|c|c|c|c|c|c|}
\hline \multirow{2}{*}{$\mathrm{Ca}(\mathrm{OH})_{2}$} & Conc./mM & 50 & 100 & 200 & 400 \\
\hline & Quant. $/ \mathrm{cm}^{3}$ & 1600 & 800 & 400 & 200 \\
\hline \multirow{2}{*}{$\mathrm{H}_{3} \mathrm{PO}_{4}$} & Conc./mM & 15 & 30 & 60 & 120 \\
\hline & Quant. $/ \mathrm{cm}^{3}$ & 3200 & 1600 & 800 & 400 \\
\hline \multicolumn{2}{|c|}{ Collagen/g } & \multicolumn{4}{|c|}{2.01} \\
\hline $\mathrm{H}_{2} \mathrm{O}$ & Quant. $/ \mathrm{cm}^{3}$ & 1600 & 800 & 400 & 200 \\
\hline
\end{tabular}

suspension is previously added to measure reaction $\mathrm{pH}$ and maintain reaction temperature from start of the reaction. Reaction temperature is controlled and maintained with the outer water bath. Reaction solution $\mathrm{pH}$ is measured with the $\mathrm{pH}$ meter equipped with electricity outlet to turn on and off power of tube pumps to control $\mathrm{pH}$ of reaction solution.

After several synthesis conditions were examined, $\mathrm{pH} 9$ and $40{ }^{\circ} \mathrm{C}$ of outer water bath temperature (reaction solution temperature of approximately $37^{\circ} \mathrm{C}$ ) were found to be the best conditions to form the HAp/Col composites. Typical transmission electron micrograph and selected area electron diffraction are shown in Fig. 4. Although the alignment of HAp nanocrystals are not complete, this level of alignment is observed in immature bone. Consequently, the authors considered that the HAp/Col has bone-like nanostructure. Chemical compositions of the HAp/Col are easily controlled by raw material ratios, i.e., calcium, phosphate and collagen ratios.

\subsection{Influence of concentration of raw materials ${ }^{14)}$}

Generally, concentration in a reaction vessel controls size of crystals formed in it. The author hypothesized that this analogue can be applied to the HAp/Col formation. The same amount of HAp/Col was synthesized under different starting material concentration. The synthesis conditions are summarized in Table 1. Obtained quantity of the HAp/Col was $10.05 \mathrm{~g}$, and HAp/Col mass ratio was $4: 1$. As described above, water amount in the reaction vessel differed according to calcium hydroxide suspension

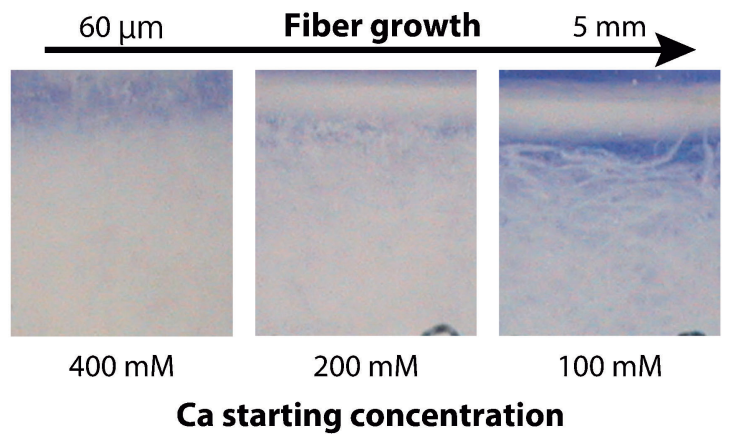

Fig. 5. HAp/Col fiber synthesized from different starting material concentrations.

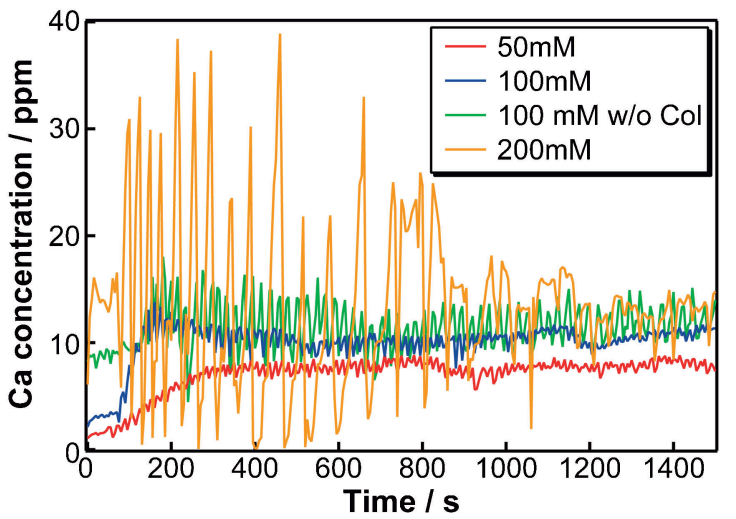

Fig. 6. Changes in calcium ion concentration during the HAp/ Col synthesis.

amount; therefore, raw material concentration in the reaction vessel is automatically changed in this system.

Photographs of the HAp/Col fibers obtained from different conditions are shown in Fig. 5. As the hypothesis, fibers, more precisely fibrous aggregates of the HAp/Col nanofibers, grow with decreasing in concentration in the reaction solution. Changes in calcium ion concentration measured with calcium ion selective electrode during the HAp/Col synthesis at 50, 100 and $200 \mathrm{mM}$ of starting Ca concentration are illustrated in Fig. 6. Starting Ca concentration of $100 \mathrm{mM}$ without adding collagen is measured as a control. As seen in Fig. 6, Ca concentrations during pseudo-plateau area, e.g., 400-1400 s, are very similar, but fluctuations of $\mathrm{Ca}$ ion concentration are quite different. Considering the standard deviation as an index of fluctuation, results are summarized in Table 2. Starting concentrations that allow long fiber growth, 50 and $100 \mathrm{mM}$, demonstrate very small fluctuations, instead standard deviation for short fiber grown conditions, $200 \mathrm{mM}$, shows half of the reaction concentration. Further, starting concentration of $100 \mathrm{mM}$ without Col demonstrates higher fluctuation than that with Col. This result suggests Col influences on HAp formation on its surface.

\subsection{Mechanism for nanostructure formation}

As the same as composite with the synthetic biodegradable polymers, the HAp/Col demonstrates an electrostatic interaction between $\mathrm{Ca}^{2+}$ on HAp and C-O 1.5 fold bond 
Table 2. Pseudo-plateau concentration (average) and standard deviation as an index of fluctuation

\begin{tabular}{ccccc}
\hline Starting Ca concentration/mM & 50 & 100 & 200 & $100 \mathrm{w} / \mathrm{o}$ Col \\
\hline Concentration/ppm & 7.69 & 10.2 & 13.0 & 11.4 \\
Standard deviation & 0.58 & 0.75 & 6.50 & 1.88 \\
\hline
\end{tabular}

of carboxyl group on $\mathrm{Col}$ in reflection FTIR. ${ }^{13)}$ Sato et al. reported that HAp is formed onto on Langmuir-Blodgett (LB) monolayer terminated by carboxyl group but not on that terminated by amino group. ${ }^{15)}$ This results suggest carboxyl group on the Col also plays a role of nucleation center for HAp nanocrystal. They also revealed that HAp crystals close to substrate LB monolayer terminated by the carboxyl groups demonstrate $c$-axis orientation along with carboxyl group alignment, ${ }^{16)}$ i.e., aligned carboxyl groups interact with $\mathrm{Ca}^{2+}$ on HAp crystals and align HAp crystals in one direction.

Carboxyl groups on Col molecule of course have alignment almost the same direction to molecule itself; therefore, HAp crystals mostly align along Col molecules in the HAp/Col. This phenomenon suggests that HAp nanocrystals in bone also grow epitaxially from solution. This means that even though precursor model, calcium hydrogen phosphate dihydrate or octacalcium phosphate was formed before HAp formation in bone, is correct, dissolution-reprecipitation process is necessary to grow HAp epitaxially as seen in bone.

\subsection{Fabrication of biomaterials}

The $\mathrm{HAp} / \mathrm{Col}$ is a fibrous nanocomposite, and simple lyophilization of the $\mathrm{HAp} / \mathrm{Col}$ gives a cotton-like soft material, therefore needs to fabricate shapes to suit for biomedical use. The first shape is dense body ${ }^{13)}$ which is shaped by compression squeezing of water from the HAp/ $\mathrm{Col}$ fiber in a specially designed mold. The dense body has a maximum 3-point flexural strength of approx. $40 \mathrm{MPa}$ and does not have enough strength for bone void fillers without a fixator. The second shape is a porous body using ice crystal grown from collagen hydrogel containing the $\mathrm{HAp} / \mathrm{Col}$ as porogens. ${ }^{17)}$ The $\mathrm{HAp} / \mathrm{Col}$ porous body (sponge) is a rigid in dry condition; however, it demonstrates sponge-like viscoelasticity in wet condition, a practical use condition, as exhibits in Fig. 7. The third one is paper-like membrane with a translucency. ${ }^{18)}$ After that, injectable self-setting paste with anti-decay property ${ }^{19)}$ and coating on metal ${ }^{20)}$ are also fabricated.

\subsection{Influences on cells in vitro}

Osteoblastic cell line, MG63 derived from human osteosarcoma, was used for cytocompatibility and influences on osteoegenic cell functions of the $\mathrm{HAp} / \mathrm{Col}$ paper-like membrane ${ }^{18)}$ and sponge. ${ }^{21)}$ The cells are well proliferated on the HAp/Col membrane as the same as the tissue culture polystyrene (TCPS) and demonstrate no difference in shape in optical microspcopic observation after Giemsa staining. Alkaline phosphatase (AlkP), an early-stage marker of osteogenesis, gene expression of MG63 cultured
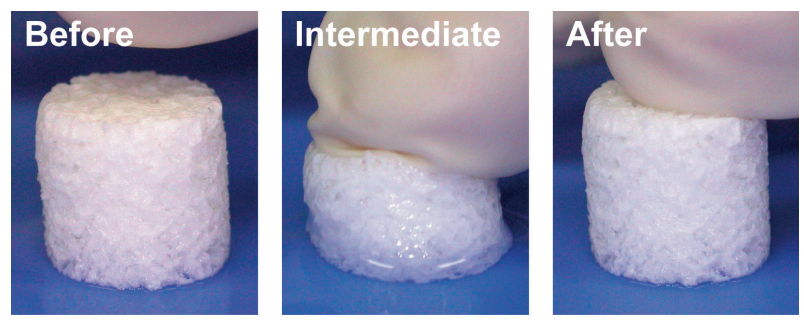

Fig. 7. Sponge-like viscoelasticity of the wet HAp/Col sponge.

on the HAp/Col membrane shows more than three times higher than that on the TCPS both with and without addition of osteogenic supplements, $50 \mu \mathrm{g} / \mathrm{ml} \mathrm{L}$-ascorbic acid phosphate magnesium salt and $10 \mathrm{nM} \beta$-glycerophosphate. ${ }^{18)}$ Pressure/perfusion culture was performed for the $\mathrm{HAp} / \mathrm{Col}$ sponge using a Col sponge as a control. ${ }^{21)}$ Initial cell proliferation on the $\mathrm{HAp} / \mathrm{Col}$ porous body indicates the similar number to that of the Col sponge, but later cell numbers on the $\mathrm{HAp} / \mathrm{Col}$ sponge are greater than those on the Col sponge. The Col sponge has a high affinity to cells and proliferates cells very well; however, exchanges of medium from inside of sponge to outside and vise-versa are limited due to its small pore size, and cells inside the sponge move out to peripheral of the Col sponge. Contrarily, the HAp/Col sponge has larger pore size, and cells migrate into inside of the HAp/Col sponge. This is the reason why the $\mathrm{HAp} / \mathrm{Col}$ demonstrates higher proliferation at latter periods. Gene expression of AlkP is greater for cells on the Col sponge at 7, 10 and 14 days after incubation, because AlkP is upregulated when the cell density is high and it is higher on the peripheral of the Col sponge than the HAp/Col sponge. Osteocalcin, the latter-stage marker, gene expression of cells on the HAp/Col and Col sponge showed no significant differences. However, cells inside of the $\mathrm{HAp} / \mathrm{Col}$ sponge only form bone nodules at 21 days after incubation. From these results, the HAp/Col improves osteogenic activity to cells in vitro.

Osteoclastic differentiation of bone marrow cells on the HAp/Col was examined by co-culture of primary osteoblasts and bone marrow cells isolated from C57BL/6 mice ${ }^{22)}$ following to Irie's paper. ${ }^{23)}$ Bone marrow cells in the method differentiate through cytokines secreted from osteoblasts upregulated by osteoclast differentiation supplements, $10 \mathrm{nM}$ of 1,25-Dihydroxyvitamin D3 and $1 \mu \mathrm{M}$ of prostaglandin E2. Bone marrow cells cultured on control groups, dentin, glutaraldehyde-crosslinked dentin, sintered HAp and TCPS differentiate after addition of the supplements and dose not differentiate without addition of them, except for slight differentiation observed for cells on dentin. The HAp/Col demonstrated osteoclastic differentiation of bone marrow cells with or without addition of the supplements. Even after gene expression analysis, the author and his colleague cannot specify the HAp/Col role to the osteoclastic differentiation of bone marrow cells. Possible roles of the HAp/Col are: 1. Absorption of osteoprotegerin, $\mathrm{OPG}$, an inhibitor of receptor activator of NF-jB ligand, RANKL, which bind to its receptor, RANK, 
of osteoclasts and their precursors to regulate osteoblastic differentiation and activation of osteoclasts, and/or 2 . Upregulation of osteoclast-associated immunoglobulinlike receptor, OSCAR, which is considered as an initiator of osteoclast differentiation, by specific collagen conformation in the HAp/Col.

\subsection{Bone tissue reactions ${ }^{13), 19), 20), 24)-33) ~}$}

The HAp/Col dense body with a several holes parallel and perpendicular to distal-proximal direction was implanted into beagle's tibial full segmental defect of $2 \mathrm{~cm}$ with external fixation. ${ }^{13)}$ At 3 months after implantation, the HAp/Col is almost disappeared. Hematoxylin-eosin, AlkP and tatrate-resistant acid phosphatase (TRAP, a marker of osteoclast) stained sections demonstrated that the disappearance of the HAp/Col is resorption by osteoclasts followed by new bone formation by osteoblasts, i.e., bone remodeling process. Actually, body fluid is supersaturated to HAp and cannot dissolve HAp, not like $\beta$ TCP, thus, HAp has to be decomposed biochemically, e.g., resorbed by osteoclasts or phagocytosed by macrophages. Hydroxyapatite particles in generally used HAp ceramics is too large to be decomposed completely by these biochemical reactions. This is the reason why HAp ceramics is practically non-biodegradable. Contrarily, HAp in the HAp/Col is a nanocrystal and can be decomposed biochemically; accordingly, the HAp/Col become the world first synthetic material that completely incorporated into bone remodeling process to substitute with new bone. The authors also revealed that resorption rate is easily controlled by crosslinking to $\mathrm{Col}$ in the $\mathrm{HAp} / \mathrm{Col}^{29}{ }^{29}$ Considering cell and tissue penetration, the HAp/Col sponge was chosen for practical use as a commercialized product. In 2013, the HAp/Col sponge, $\mathrm{ReFit}^{\circledR}$, was start to be sold from HOYA Technosurgical Co. The HAp/Col sponge demonstrates excellent bone substitution property in bones in its clinical trial ${ }^{34)}$ and practical use. Further, earlier bone formation of the $\mathrm{HAp} / \mathrm{Col}$ for anterior cervical discectomy and fusion using Ti cage than $\beta$-TCP is also reported. ${ }^{35)}$

Recently, the authors found that Ti dip-coated with the HAp/Col implanted into subperiosteum of rat cranium induces trice faster osseointegration (gaps between material and bone are not observed with naked-eye and optical microscope but observed with electron microscope) than pure and biomimetic HAp coated $\mathrm{Ti}^{20}{ }^{20}$ The detailed mechanism is under investigation but it will be applied to dental and medical prostheses.

The authors, as mentioned above, developed injectable self-setting HAp/Col paste with anti-decay property using (3-glycidoxypropyl)trimethoxysilane. ${ }^{19)}$ The HAp/Col paste shows no local and systemic toxic effect including irregular inflammation after direct injection to tibial hole of pigs. At 3 month postimplantation, the $\mathrm{HAp} / \mathrm{Col}$ paste is completely substituted with newly formed bone.

\section{Summary}

The author has focused on interfacial interaction between inorganic and organic substances to fabricate bone regenerating materials based on calcium phosphate ceramics in collaboration with many colleagues in material science, biochemical, veterinary, medical and dental fields. This concept allows high interconnected porous and porosity ceramics with sufficient mechanical strength for bone void fillers, thermoplastic and biodegradable composite with sufficient mechanical properties for GBR treatment, and bone-like nanocomposite of HAp and Col which is completely incorporated into bone remodeling process. The HAp/Col demonstrates many potentials to use for bone void filler in porous and injectable paste, coating on metal to accelerate osseointegration. These researches and developed materials contribute to human health.

Acknowledgement The author expresses deep appreciate to co-authors in the referred papers, especially Prof. Emeritus Junzo Tanaka, Drs. Yasushi Suetsugu, Sun-Baek Cho, Kimiyasu Sato, Ms. Ikuko Akahane in the same group in NIMS with the author at that time, and Profs. Hiroo Miyairi, Kenichi Shinomiya, Kazuo Takakuda, Keiji Moriyama, Soichiro Itoh, Yoshihisa Koyama, Shinichi Sotome, Masayoshi Uezono of Tokyo Medical and Dental University. The author also thanks to all students researched under my guidance. The studies in the paper were supported in part by CREST, JST and Research Promotion Bureau, Ministry of Education, Culture, Sports, Science and Technology, Japan under the contract No.17-83. Lastly, the author dedicates the paper to animals sacrificed for the researches described in.

\section{References}

1) M. Kikuchi, Y. Suetsugu, J. Tanaka, K. Imura, H. Umemoto, N. Ninowa, M. Kinoshita, A. Hojo and H. Yamazaki, "Calcium phosphate porous sintered body and production thereof", JP3400740, US6,340,648, GB2348872A.

2) Y. Suetsugu, Y. Hotta, M. Iwasashi, M. Sakane, M. Kikuchi, T. Ikoma, T. Higaki, N. Ochiai and J. Tanaka, Key Eng. Mat., 330-332, 1003-1006 (2007).

3) W. Bonfiled, Ann. N. Y. Acad. Sci., 523, 173-177 (1998).

4) https://medtech.pharmaintelligence.informa.com/ MT087959/Smith-and-Nephew-Richards-800th-Hapexmiddle-ear-implant, visited 17th April (2020).

5) M. Kikuchi, Y. Koyama, T. Yamada, Y. Imamura, T. Okada, N. Shirahama, K. Akita, K. Takakuda and J. Tanaka, Biomate., 25, 5979-5986 (2004).

6) M. Kikuchi, Y. Suetsugu, J. Tanaka and M. Akao, J. Mater. Sci. Mater. Med., 8, 361-364 (1997).

7) M. Kikuchi and J. Tanaka, J. Ceram. Soc. Jpn., 108, 642-645 (2000).

8) M. Kikuchi, Y. Koyama, K. Takakuda, H. Miyairi, N. Shirahama and J. Tanaka, J. Biomed. Mater. Res., 62, 265-272 (2002).

9) M. Kikuchi, J. Tanaka, Y. Koyama and K. Takakuda, J. Biomed. Mater. Res. B, 48, 108-110 (1999).

10) S.-B. Cho, M. Kikuchi, Y. Suetsugu and J. Tanaka, Key Eng. Mat., 132-136, 802-805 (1997).

11) C. Du, F. Z. Cui, Q. L. Feng, X. D. Zhu and K. de Groot, J. Biomed. Mater. Res., 42, 540-548 (1998).

12) M. J. Olszta, X. Cheng, S. S. Jee, R. Kumar, Y.-Y. Kim, M. J. Kaufman, E. P. Douglas and L. B. Gower, Mater. 
Sci. Eng., R, 58, 77-116 (2007).

13) M. Kikuchi, S. Itoh, S. Ichinose, K. Shinomiya and J. Tanaka, Biomaterials, 22, 1705-1711 (2001).

14) M. Kikuchi, S. Itoh, H. N. Matsumoto, Y. Koyama, K. Takakuda, K. Shinomiya and J. Tanaka, Key Eng. Mat., 240-242, 567-570 (2003).

15) K. Sato, Y. Kumagai and J. Tanaka, J. Biomed. Mater. Res., 50, 16-20 (2000).

16) K. Sato, T. Kogure, Y. Kumagai and J. Tanaka, J. Colloid Interf. Sci., 240, 133-138 (2001).

17) M. Kikuchi, T. Ikoma, D. Syoji, H. N. Matsumoto, Y. Koyama, S. Itoh, K. Takakuda, K. Shinomiya and J. Tanaka, Key Eng. Mat., 254-256, 561-564 (2004).

18) M. Kikuchi, Key Eng. Mat., 330-332, 313-316 (2007).

19) T. Sato, Y. Shirosaki, M. Nagaya, Y. Asano, K. Nakano, H. Nagashima, M. Aizawa and M. Kikuchi, J. Asian Ceram. Soc., 6, 322-331 (2018).

20) M. Uezono, K. Takakuda, M. Kikuchi, S. Suzuki and K. Moriyama, J. Biomed. Mater. Res. B, 101B, 1031-1038 (2013).

21) T. Yoshida, M. Kikuchi, Y. Koyama and K. Takakuda, J. Mater. Sci. Mater. Med., 21, 1263-1272 (2010).

22) M. Kikuchi and A. Irie, Key Eng. Mat., 396-398, 449452 (2009).

23) A. Irie, M. Takami, H. Kubo, N. Sekino-Suzuki, K. Kasahara and Y. Sanai, Bone, 41, 165-174 (2007).

24) S. Itoh, M. Kikuchi, K. Takakuda, Y. Koyama, H. N. Matsumoto, S. Ichinose, J. Tanaka, T. Kawauchi and K. Shinomiya, J. Biomed. Mater. Res., 54, 445-453 (2001).

25) S. Itoh, M. Kikuchi, K. Takakuda, K. Nagaoka, Y. Koyama, J. Tanaka and K. Shinomiya, J. Biomed.
Mater. Res., 63, 507-515 (2002).

26) S. Itoh, M. Kikuchi, Y. Koyama, K. Takakuda, K. Shinomiya and J. Tanaka, Biomaterials, 23, 3919-3926 (2002).

27) S. Sotome, T. Uemura, M. Kikuchi, C. Jian, S. Itoh, J. Tanaka, T. Tateishi and K. Shinomiya, Mater. Sci. Eng., C, 24, 341-347 (2004).

28) S. Itoh, M. Kikuchi, Y. Koyama, K. Takakuda, K. Shinomiya and J. Tanaka, Cell Transplant., 13, 451-461 (2004).

29) M. Kikuchi, H. N. Matsumoto, T. Yamada, Y. Koyama, K. Takakuda and J. Tanaka, Biomater, 25, 63-69 (2004).

30) S. Itoh, M. Kikuchi, Y. Koyama, H. N. Matsumoto, K. Takakuda, K. Shinomiya and J. Tanaka, Biomed. Mater. Eng., 15, 29-41 (2005).

31) T. Nishikawa, K. Masuno, K. Tominaga, Y. Koyama, T. Yamada, K. Takakuda, M. Kikuchi, J. Tanaka and A. Tanaka, Implant Dent., 14, 252-260 (2005).

32) A. Tsuchiya, S. Sotome, Y. Asou, M. Kikuchi, Y. Koyama, T. Ogawa, J. Tanaka and K. Shinomiya, J. Med. Dent. Sci., 55, 91-99 (2008).

33) T. Hiratsuka, M. Uezono, K. Takakuda, M. Kikuchi, S. Oshima, T. Sato, S. Suzuki and K. Moriyama, J. Biomed. Mater. Res., 108B, 391-398 (2020).

34) S. Sotome, K. Ae, A. Okawa, M. Ishizuki, H. Morioka, S. Matsumoto, T. Nakamura, S. Abe, Y. Beppu and K. Shinomiya, J. Orthop. Sci., 21, 373-380 (2016).

35) N. Kikuchi, Y. Ohara, Y. Tomita, H. Matsuoka and J. Mizuno, Spinal Surg., 31, 257-261 (2017).

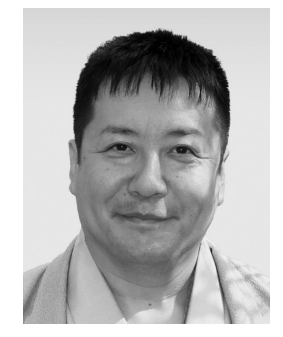

Masanori Kikuchi is a Group Leader of Bioceramics Group, National Institute for Materials Science (NIMS) in Tsukuba, Japan from 2007. He received his B.E. (1990), M.E. (1992) and Ph.D. (1995) from Waseda University, Japan. He started his job in 1995 as a Visiting Researcher of Waseda University, then moved and has worked at National Institute for Research in Inorganic Materials (NIRIM, presently NIMS) from 1995. He also worked on international standardization from 2004 and is currently a chairperson of the Japanese committee of ISO/TC 150 from 2020 and a convener of ISO/TC 150/SC 1/WG 3 from 2011. He established the first international standard for tissue engineering medical products, ISO 19090. He also has worked as a Visiting Professor of Hokkaido University from 2011 and Professor of University of Tsukuba from 2016. 